\title{
Warum Säuglinge anders schlafen
}

Herbert Renz-Polster

Das Schlafen ist ein menschliches Grundbedürfnis von Anfang an. Doch Säuglinge schlafen anders als Erwachsene. Kein Wunder, dass am Babyschlaf oft Konflikte in jungen Familien aufkeimen: Dürfen Babys ins Elternbett? Müssen sie das Durchschlafen antrainieren? Was ist normal? Diese Fragen beantwortet Dr. med. Herbert Renz Polster beim Forum Hebammenarbeit in Wiesbaden am 14. November 2020. Eine Vorschau auf seinen Vortrag mit Gedanken, die auch für die Beratung junger Eltern hilfreich sein können.

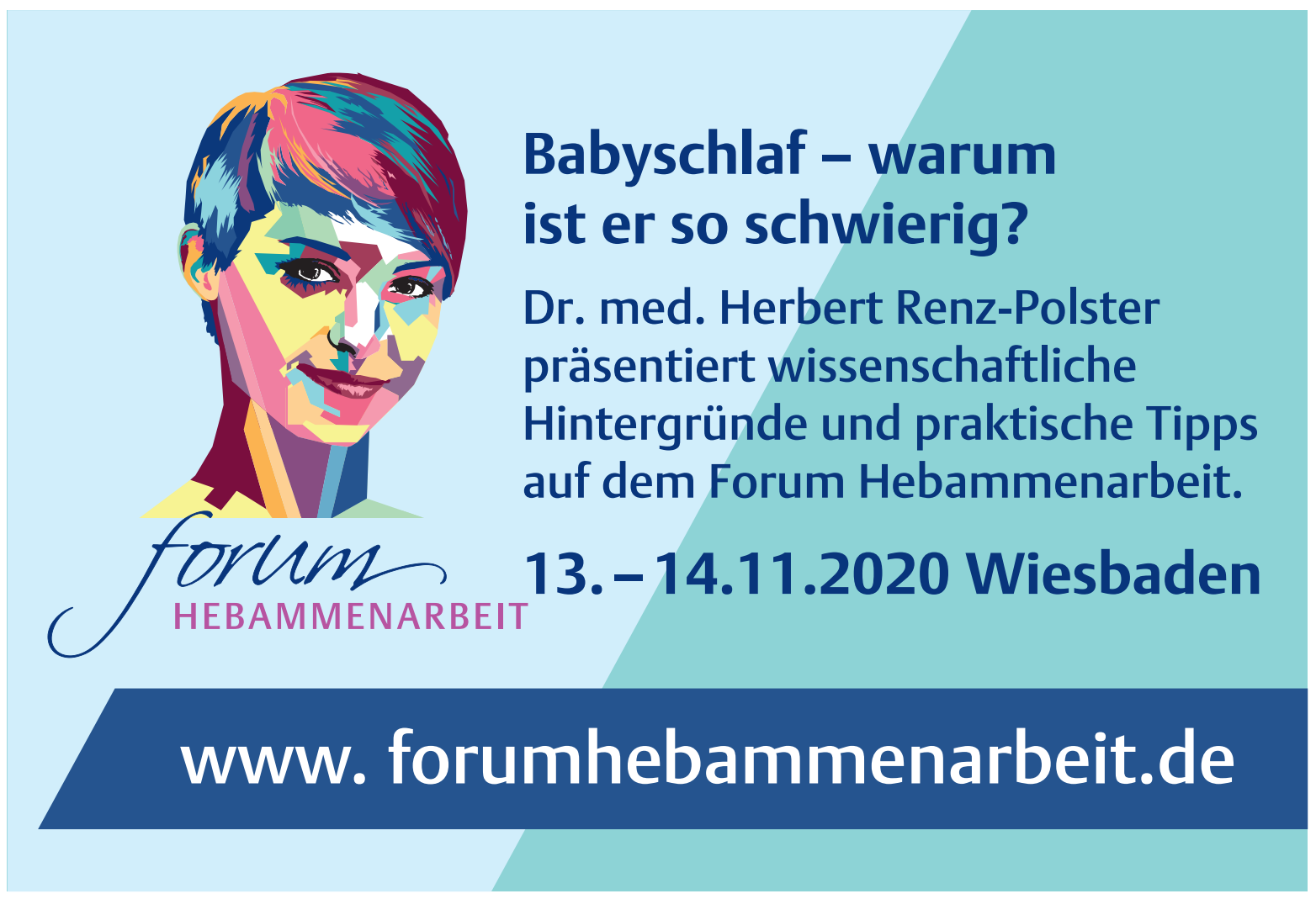

\section{Was ist normal?}

So mancher Auseinandersetzung in Eltern- und Fachkreisen ließe sich womöglich aus dem Wege gehen, würden sich einige grundlegende Übereinkünfte treffen: Zum Beispiel aus der Erkenntnis heraus, dass es „den“ Babyschlaf an sich nicht gibt. Oder dass ein Kind nicht deswegen „normal“ ist, weil es zu bestimmten Uhrzeiten ein- bzw. ab einem bestimmten Alter zuverlässig durchschläft. Und überhaupt: Dass der Schlaf so individuell ist wie alle anderen Verhaltensweisen von Kindern. Warum sollten sie ausgerechnet dabei gleich sein? Den Eltern kann also getrost zu mehr Gelassenheit geraten werden: Dass Kinder in den ersten drei Lebensjahren regelmäßig und dauerhaft durchschlafen, ist eher die Ausnahme als die Regel. 


\section{Die Schlafdauer}

Der Spielraum ist riesengroß: Als Neugeborene schlafen manche Säuglinge nur rund 11, andere hingegen ganze 20 Stunden pro Tag. Im Mittel liegen sie bei 14,5 Stunden. Mit 6 Monaten schlafen sie durchschnittlich immerhin noch 13 Stunden. Doch die Unterschiede bleiben groß: Manche Babys kommen mit 9 Stunden aus, andere brauchen dagegen bis zu 17 Stunden Schlaf pro Tag. Im zweiten Lebensjahr liegt der tägliche Schlafbedarf im Schnitt bei 12 Stunden, plus/minus 2 Stunden. Mit 5 Jahren genügen manchen Kleinkindern 9 Stunden, andere brauchen noch immer 14 . Genauso wie manche Kinder nach dem Stillen länger satt sind als andere, ist es offenbar auch mit dem Schlafen.

\section{Die Schlafhäppchen}

Während sich der Schlaf des Neugeborenen gleichmäßig über Tag und Nacht verteilt, lässt sich später ein Muster erkennen: Etwa ab dem 2. bis 3. Monat wickeln Säuglinge einen immer größeren Teil ihres Schlafs in der Nacht ab. Trotzdem halten die meisten mit 5 bis 6 Monaten immer noch etwa 3 Tagesschläfchen. Wenige Monate später kommen viele von ihnen dann tagsüber schon mit 2 Schlafportionen aus. Und sobald sie laufen können, begnügen sich viele von ihnen-aber eben längst nicht alle - mit einem einzigen Mittagsschlaf. Und mit 4, spätestens 5 Jahren ist auch dieser bei den allermeisten Kindern nicht mehr angesagt.

\section{Mythos Durchschlafen}

Die Erfahrung zeigt: Es ist sehr selten, dass ein Baby die ganze Nacht ohne Pause schläft. In der Wissenschaft zählt ein Baby dann zu den sog. Durchschläfern, wenn es nach Elternangaben von 0 bis 5 Uhr nicht nach ihnen verlangt. Im ersten Lebenshalbjahr wachen (nach Elternangaben) $86 \%$ der Säuglinge regelmäßig nachts auf. Etwa ein Viertel davon sogar 3-mal und öfter. Zwischen 13 und 18 Monaten wachen noch immer zwei Drittel der Kleinkinder regelmäßig nachts auf, Jungs insgesamt häufiger als Mädchen. Auch Babys im Elternbett melden sich häufiger, dafür aber kürzer. Gestillte Kinder sind mit dem Durchschlafen insgesamt später dran als nicht gestillte Kinder.

Auch Wissenschaftler untersuchen das Phänomen: Zeichnet man den Säuglingsschlaf mit der Videokamera auf, so werden 3 Monate alte Kinder im Schnitt nachts 2- bis 3-mal wach. Mit 9 Monaten wachen sie 5-mal pro Nacht auf. Und mit 12 Monaten sind sie wieder bei 2- bis 3-mal pro Nacht. Selbst im Kleinkindalter ist der Nachtschlaf noch nicht von Dauer: Über ein Drittel der Kinder meldet sich nachts noch mit zweieinhalb Jahren regelmäßig.

Wann Kinder durchschlafen, hängt sowohl von ihrem Temperament als auch davon ab, was in ihrem Leben gerade vorgeht. Oft wird das Durchschlafen mit dem
Beginn der Fremdelphase ( 8 bis 11 Monate) wieder schwieriger. Auch mit etwa 3 bis 4 Jahren wachen viele Kinder nachts wieder häufiger auf.

Das nächtliche Aufwachen scheint zu den unvermeidlichen Begleiterscheinungen des kindlichen Schlafs zu gehören. Die Schlafforschung definiert aus diesem Grund das Durchschlafen anders als viele Eltern:

Merke

Durchschlafen heißt nicht, dass ein Kind die ganze Nacht schläft. Ein Säugling schläft vielmehr dann durch, wenn er nachts 6 bis 8 Stunden lang Ruhe gibtd. h. wenn es ihm gelingt, nach den Wachphasen wieder von alleine in den Schlaf zu finden.

\section{Warum Kinderschlaf anders ist}

Der Schlaf des Säuglings ist unruhiger als der des Erwachsenen. Betrachtet man ihn aus dem Blickwinkel der evolutionären Verhaltensbiologie, ergibt sich rasch eine mögliche Erklärung: Kein Lebewesen wird unreifer geboren als das Menschenkind. Der im Vergleich zu anderen Säugetieren ungeheuer große Kopf passt nun einmal nur in einer Kleinausgabe durch den engen Geburtskanal einer zweibeinigen Mutter. Ein Großteil der Entwicklung des Gehirns erfolgt beim Menschen daher erst in der Zeit nach der Geburt. Tatsächlich wächst das Gehirn in den ersten drei Lebensjahren ungeheuer rasant auf die 2- bis 3-fache Größe an.

Bei diesem Entwicklungsspurt läuft das Gehirn natürlich nicht im Schonbetrieb-noch nicht einmal im Schlaf. Denn anders als Erwachsene schalten die Kleinen ihr Gehirn im Schlaf nur ungern herunter. Sie verbringen vielmehr große Teile ihres Schlummers in einer Art Reifungsschlaf. In dieser Zeit ordnet und sortiert das Gehirn Erlebnisse, Eindrücke und Gefühle und verfestigt damit das Gelernte. Der ganze Körper bleibt dabei aktiv, was sich auch daran zeigt, dass die Augen hinter den Lidern oft hin- und herspringen. Ein Teil dieses aktiven Schlafs wird deshalb auch als REM-Schlaf (Rapid eye movement sleep) bezeichnet. Während dieses aktiven Entwicklungsschlafs sind die Kinder leichter erweckbar als während der anderen Anteile des Schlafs.

Die Gehirnentwicklung in dieser kurzen Zeit auf genanntes Maß voranzubringen, kostet zudem sehr viel Energie. Denn Nerven- bzw. Gehirnzellen nutzen, anders als normale Körperzellen, fast ausschließlich Zucker als Energielieferant. Muttermilch enthält fast doppelt so viel Milchzucker wie Kuhmilch. In dieser Aufholphase melden sich die Kleinen daher entsprechend häufig zum „Nachtanken" - und das leider auch im Schlaf. Der Milchzucker wird rasch ins Blut aufgenommen und verwertet. 


\section{Soll das Kind nachts gestillt werden?}

Auf diese Frage gibt es sehr unterschiedliche Antworten. Viele davon sind willkürlich und wissenschaftlich nicht belegt. Früher galt z. B. die „nächtliche Stillpause“ von 8 Stunden als Norm-von Anfang an. Noch immer wird (teils sogar von Kinderärzten) behauptet, Babys bräuchten schon ab 8 Wochen keine nächtlichen Mahlzeiten mehr. Stimmte das, würden Eltern öfter ungestört schlafen können. Es gibt allerdings gute Argumente, mit denen sich diese Aussagen widerlegen lassen. Sie setzen ja im Grunde den Rhythmus der Nahrungsaufnahme eines Säuglings ab dem 7. Lebensmonat mit dem eines Erwachsenen gleich. Richtig ist vielmehr, dass nächtliches Aufwachen eine normale, zu erwartende Reaktion von Säuglingen und kleinen Kindern ist, denn:

- Auch in der zweiten Hälfte des ersten Lebensjahres wachsen Säuglinge noch so schnell, dass sie ihr Körpergewicht in diesem Zeitraum fast verdoppeln.

- Wegen ihres raschen Hirnwachstums und ihres aktiveren Schlafes brauchen Kleinkinder in den ersten 2 bis 3 Jahren, auf ihr Körpergewicht bezogen, etwa 4mal mehr Kalorien als Erwachsene.

- Kleine Kinder unterscheiden sich individuell enorm in ihrer Futterverwertung: Manche brauchen zum Wachsen fast doppelt so viele Kalorien wie andereschon das spricht gegen feste Angaben von Zeiträumen und Stillpausen.

- Die kindliche Entwicklung verläuft oft in Schüben, was ebenfalls gegen genaue Monatsangaben spricht.

- Zudem unterscheiden sich gestillte und mit der Flasche gefütterte Kinder in ihrem nächtlichen Nahrungsbedarf. Stillkinder melden sich insgesamt häufiger, vielleicht weil Muttermilch rascher verdaut wird.

Selbst der Schlafort beeinflusst den nächtlichen Nahrungsbedarf. So nehmen bei ihrer Mutter schlafende gestillte Säuglinge nachts immerhin ein Drittel mehr Kalorien zu sich als die im eigenen Bett schlafenden gestillten Kinder. Das könnte damit zu tun haben, dass Kinder im Nahbereich ihrer Mutter höhere Anteile an aktiven Schlafphasen haben.

Die Angabe vom 4. bis 6. Monat als Beginn des nahrungsfreien Durchschlafens wird manchmal damit begründet, dass das Baby von da an Beikost bekomme und durch die festere Nahrung mehr Kalorienreserven habe. Die Tatsache, dass Beikost im Vergleich zur Muttermilch nicht sättigender, sondern sogar kalorienärmer ist, schwächt jedoch auch diese These.

\section{Merke}

Aus evolutionsbiologischer Sicht ist der Schlaf des kleinen Kindes deshalb so leicht geknüpft, um ihm das Trinken an der Brust zu ermöglichen und den reifungsbedingt großen Nährstoffbedarf zu decken. Durch- schlafen sagt nichts über den Entwicklungsstand eines Kindes aus. Kinder, die mit sechs Monaten durchschlafen, unterscheiden sich weder in ihrer psychoemotionalen Reife noch in sonstigen Lebenskompetenzen von denen, die es nicht tun.

\section{Kinder den Schlaf finden lassen}

Aus Sicht der Evolution betrachtet, müssen Kinder zwar nicht erst das Schlafen lernen. Wir dürfen erwarten, dass sie so etwas Elementares und Lebenswichtiges von Natur aus können. Allerdings gilt dies mit einer wichtigen Einschränkung - die Schlafbedingungen müssen stimmen:

- Erste Bedingung ist Entspannung. Alle Säugetiere schlafen nur, wenn sie sich nicht bedroht fühlen. Adrenerge Stressreaktionen verhindern den Schlaf zuverlässig. Das gilt auch für Kinder: Um in den Schlaf zu finden, muss ein Kind nicht nur müde, satt und warm sein, sondern auch frei von Angst. Es muss sich geschützt und geborgen fühlen.

- Zweite Bedingung ist Mitbestimmung. Wenn Kinder müde werden, so tun sie das in Wellen: Etwa alle 50 Minuten tritt ein kleines Kind tagsüber von einer aktiveren Phase in eine beruhigte Phase ein. Dann öffnet sich das Tor zum Einschlafen. Bekommt es mit einer Müdigkeitswelle nicht die Kurve in den Schlaf, ist es nach wenigen Minuten schon wieder munter. Kann das Kind selbst bestimmen, wann es auf den Schlafzug aufspringen will, so sucht es sich in seiner „Tiefphase“ vielleicht die Brust der Mutter oder die Nähe einer sonstigen vertrauten Person. Das erklärt, warum die mütterliche Brust als Einschlafhilfe so gut wirkt: Kinder spüren die schützende Nähe der Mutter, ihre Rhythmen sind sinnlich erfahrbar, von der Atmung bis zum Herzschlag. Außerdem ist das Baby warm, bald auch satt und es kann den Zeitpunkt selbst bestimmen, wann es von der Schläfrigkeit in den Schlaf abtaucht.

\section{Beschützt einschlafen}

Das Dilemma für Eltern liegt auf der Hand: Ihr nächtliches Ruhebedürfnis ist schwer vereinbar mit den beschriebenen Bedingungen. Denn die Entspannung und Mitbestimmung des Kindes setzt die Anwesenheit eines vertrauten Erwachsenen voraus. So unpraktisch dies sein mag, evolutionsbiologisch betrachtet ist es sinnvoll: Ohne den Schutz von Erwachsenen einzuschlafen, führte unter den Bedingungen der menschlichen Frühgeschichte in den sicheren Tod. Die ungeschützten Säuglinge wären von Hyänen verschleppt, von Bären gefressen oder durch eine plötzliche Kaltfront unterkühlt worden. Kein Wunder also, dass ein vertrauter Mensch und die mit ihm verbundenen Merkmale - vom Hautkontakt bis zur gewohnten Stimme-besser beim Einschlafen helfen als ein Gutenachtlied alleine. 
Der ernüchternde Befund der Evolutionsbiologie lautet also, dass kleine Kinder keine selbstständigen Schläfer sind, sondern beschützt einschlafen wollen. Kleine Kinder lernen von sich aus Laufen und sie werden von sich aus sauber, aber sie werden nicht aufgrund eines natürlichen Programms zu selbstständigen Schläfern. Der Weg, auf dem Kinder selbstständig werden, ist vielmehr ein indirekter Weg, zu dem sowohl die Erfahrung elterlicher Nähe als auch die Erfahrung der Selbstwirksamkeit durch das Kind gehören. Explorationstrieb und Selbstwirksamkeit sind dabei beim Kind eng mit der Erfahrung emotionaler Sicherheit verbunden.

\section{Merke}

Nur wenn sich ein Kind sicher, stressfrei und wohl fühlt, kann es sich der Welt zuwenden und Schritte zur Autonomie unternehmen. Das gilt auch auf dem Weg zum selbstständigen Einschlafen. Selbstständigkeit kann jedoch nicht durch Eltern einfach anerzogen werden.

\section{Was wir Eltern raten können}

Was den Schlaf anbetrifft, geht es für Eltern und Kind darum, gemeinsam Entspannung zu finden. Denn nur damit gelingt der Schlaf. Und so individuell wie der Schlaf selbst ist auch der Weg zur Entspannung. Daher verwundert es nicht, dass es an dieser Stelle kein Patentrezept gibt. Jede Familie muss ihren Weg finden, also sich und ihr Baby kennenlernen.

Dabei können Hebammen den Eltern folgende Gedanken mitgeben:

- Keine Angst vor Nähe haben: Dass Eltern ihre Babys beim Schlafen bei sich haben, bedeutet noch lange nicht, dass die Kinder deshalb verwöhnt würden oder später nicht selbstständig werden könnten. Eine pauschale Warnung vor dem Schlafen mit Babys aus Sicherheitsgründen halte ich für unbegründet und sie ist auch unter Forschern umstritten. Solange Eltern die Grundregeln eines sicheren Schlafumfelds beachten, ist der gemeinsame Schlaf im Elternbett eine sichere Wahl.

- Individualität anerkennen: Kinder sind verschieden und sollten nicht an vermeintlichen Normen gemessen werden, denen sie gar nicht entsprechen können - weil sie eben verschieden sind.
- Kindern Zeit geben: Säuglinge brauchen eine Weile, um einen Rhythmus zu finden. Neugeborene verteilen den Schlaf gleichmäßig über Tag und Nacht, ab 2 bis 3 Monaten schlafen Säuglinge einen immer größeren Teil ihres Schlafs in der Nacht. Trotzdem brauchen die meisten mit 5 bis 6 Monaten tagsüber noch etwa 3, später nur noch 2 Schlafportionen.

- Schlafen und Stillen: Gestillte Kinder im Elternbett werden nachts zwar etwas häufiger wach, schlafen dann aber wieder rascher ein.

- Gelassen bleiben: Und sich nicht von anderen Eltern verunsichern lassen, deren Kinder vermeintlich „schon weiter“ sind. Dass alle Kinder ab einem bestimmten Alter durchschlafen und dass alles andere ungesund oder unnormal sei, ist ein Mythos und lässt sich wissenschaftlich bis heute nicht belegen.

\section{Autorinnen / Autoren}

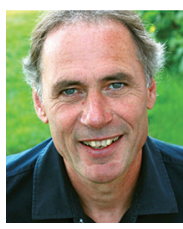

Dr. med. Herbert Renz-Polster ist Kinderarzt, Fachautor und Wissenschaftler. Er befasst sich seit vielen Jahren mit Fragen der Kindergesundheit und kindlichen Entwicklung. Zu seinen Schwerpunktthemen zählt der gesunde Schlaf von Säuglingen und Kleinkindern.

Am 14. November 2020 referiert Dr. Renz-Polster beim Forum Hebammenarbeit in Wiesbaden. Anschließend besteht die Möglichkeit, Fragen zu stellen und zu diskutieren. Programm $\rightarrow$ www.forumhebammenarbeit.de

\section{Korrespondenzadresse}

Dr. med. Herbert Renz-Polster

Rohrmoos 10

88267 Vogt

E-Mail: Herbert.Renz-Polster@posteo.de

\section{Bibliografie}

DOI https://doi.org/10.1055/a-1213-8072

Die Hebamme 2020; 33: 46-49

(c) Georg Thieme Verlag KG Stuttgart · New York

ISSN 0932-8122 